\title{
AN APPLICATION OF A $(1,2)$ QUATERNARY CORRESPONDENCE TO THE WEDDLE AND KUMMER SURFACES*
}

BY

\author{
VIRGIL SNYDER
}

Introduction.

The three-dimensional non-linear involution determined by a system of quadrics having three linear parameters has been extensively discussed. In the case in which all the quadrics have six basis points the system furnishes a convenient method of mapping the Weddle surface upon the Kummer surface. In the following paper I develop the details of this transformation analytically, obtaining a number of results already known, but by new methods, and apply the transformation to obtain a number of series of transformations which leave the surfaces invariant. A number of new transformations appear when the basis points are in simple or multiple involution. The relations which must exist among the coefficients of the equations to insure involution, the positions of the new lines lying on the Weddle surface, and the corresponding specializations of the Kummer surface are obtained.

\section{§1. The Involution $I$.}

1. Through six arbitrary points in space can be passed a linear system of quadric surfaces having three parameters. The equation of the system has the form

$$
H \equiv \lambda_{1} H_{1}+\lambda_{2} H_{2}+\lambda_{3} H_{3}+\lambda_{4} H_{4}=0 \text {. }
$$

If the coördinates $\left(\xi^{\prime}\right)$ of a point $P^{\prime}$ are substituted in $H$, between $H(x)$ and $H\left(\xi^{\prime}\right)$ one parameter, as $\lambda_{4}$, may be eliminated, and thus the net

$$
\lambda_{1}\left(H_{1} \cdot H_{4}^{\prime}-H_{1}^{\prime} \cdot H_{4}\right)+\lambda_{2}\left(H_{2} \cdot H_{4}^{\prime}-H_{2}^{\prime} \cdot H_{4}\right)+\lambda_{3}\left(H_{3} \cdot H_{4}^{\prime}-H_{3}^{\prime} \cdot H_{4}\right)=0
$$

is obtained. The coefficients of the $\lambda_{i}$ define three quadrics which intersect in eight points; as seven of these points are known the coördinates of the eighth, $P^{\prime \prime} \equiv\left(\xi^{\prime \prime}\right)$, can be expressed as rational functions of the coördinates of the six fixed points and $\left(\xi^{\prime}\right)$. In other words, the equations

* Presented to the Society December 28, 1910. 
yield the rational solutions

$$
\frac{H_{1}^{\prime \prime}}{H_{1}^{\prime}}=\frac{H_{2}^{\prime \prime}}{H_{2}^{\prime}}=\frac{H_{3}^{\prime \prime}}{H_{3}^{\prime}}=\frac{H_{4}^{\prime \prime}}{H_{4}^{\prime}}
$$

$$
\xi_{i}^{\prime \prime}=\phi_{i}\left(\xi_{1}^{\prime}, \xi_{2}^{\prime}, \xi_{3}^{\prime}, \xi_{4}^{\prime}\right) \text {. }
$$

The seventh and eighth basis points of any net of quadrics contained in a linear system having six fixed basis points define an involutorial birational transformation.*

Without restricting the problem, the basis points may be taken at the vertices of the tetrahedron of reference, the unit point and the point $(a)$. It will be convenient to designate the points by number, as follows:

$$
\begin{gathered}
(1,0,0,0) \equiv 1, \quad\left(a_{1} a_{2} a_{3} a_{4}\right) \equiv 2, \quad(0,1,0,0) \equiv 3, \quad(1,1,1,1) \equiv 4, \\
(0,0,1,0) \equiv 5, \quad(0,0,0,1) \equiv 6 .
\end{gathered}
$$

In the net defined by $\left.1,2, \ldots, 6,\left(\xi^{\prime}\right), \xi^{\prime \prime}\right)$, the line $\left(\xi^{\prime} \xi^{\prime \prime}\right)$ determines a pencil of quadrics whose basis curve consists of this line and the cubic curve $\gamma_{3}$ passing through $1,2, \ldots, 6$. The line $\left(\xi^{\prime} \xi^{\prime \prime}\right)$ is a bisecant of the cubic curve.

Any two associated points $\left(\xi^{\prime}\right),\left(\xi^{\prime \prime}\right)$ lie on a bisecant of the cubic curce $\gamma_{3}$ defined by the six basis points. There are fifteen special bundles, each defined by the points $1,2, \ldots, 6$ and a line joining some two of them. These bundles will be designated by the symbols $\{i k\}$. Similarly, if $P^{\prime}$ is on $\gamma_{3}$, the net will contain $\gamma_{3}$. It will be called $\left\{\gamma_{3}\right\}$.

2. A line $l$ (or any three points upon it) will uniquely define a quadric $H$ which contains it. When $\left(\xi^{\prime}\right)$ describes $l,\left(\xi^{\prime \prime}\right)$ will describe some curve upon $H_{l}$, always lying on the bisecant of $\gamma_{3}$ through $\left(\xi^{\prime}\right)$. The bisecants of $\gamma_{3}$ from points on $l$ define a ruled surface $R_{4}$ of order four, having $l$ for simple directrix and $\gamma_{3}$ for double curve. $R_{4}$ and $H_{l}$ intersect in a curve of order eight, composed of $l$ and a curve $c_{7}$, of order seven, the locus of $\left(\xi^{\prime \prime}\right)$. A plane through $l$ cuts $H_{l}$ in a straight line and cuts $R_{4}$ in three straight lines. The points of intersection are on $c_{7}$, hence $l$ cuts $c_{7}$ in four points. By the involutorial transformation I straight lines go into space curves of order seven, each cutting its image line in four points. Planes go into surfaces of order seven.

3. Since $R_{4}$ has $1,2, \ldots, 6$ for double points and $H_{l}$ passes through each, $c_{7}$ has six double points which are principal points of the transformation, the image of each being a quadric cone having its vertex at its associated point and containing $\gamma_{3}$ and five lines $(i k)$. These cones, each counted twice, constitute

* Geiser : Ueber zwei geometrische Probleme, Crelle's Journal, vol. 67 (1867), pp. 78-95; EBERHARDT : Ueber eine räumlich involutorische Verwandtschaft \%. Grades und ihre Kernfläche 4. Ordnung, Breslan dissertation (1885), 61 pp.; STURM: Die Lehre von den geometrischen Verwandtschaften, vol. 4 (1909), pp. 414-418. For the literature on the system of surfaces see also Staude: Encyklopädie der mathematischen Wissenschaften, III C2, $\& 141$ (p. 252). 
the entire Jacobian of the system $\phi$. The curve $\gamma_{3}$ and the lines (ik) are principal curves of the second kind, such that the image of a point on any one of them is the whole line on which it lies.

4. A plane $\pi$ yoes into a surface $F_{7}$ of order seven. Since $\pi$ has three points on $\gamma_{3}, F_{7}$ has $\gamma_{3}$ as a threefold curve, and similarly, $F_{7}$ contains each of the lines $(i k)$. Two planes intersect in $l$; their image surfaces intersect in $c_{7}$ the image of $l$, in $\gamma_{3}$ counted nine times and in the fifteen lines $(i k)$. Hence there are no further principal points in the transformation.

5. If $l$ passes through a basis point $i$, the principal cone $K_{2}^{(i)}$ is part of its image. If $l$ is the intersection of two planes $\pi, \pi^{\prime}$, each passing through $i$, the image $c_{7}$ will be the intersection of $F_{7}, F_{7}^{\prime}$. Both these surfaces contain $k_{2}(i)$ as factor. Each of the residual quintics contains $\gamma_{3}$ as double curve and the ten lines $(l k)$ not passing through $i$. Thus the proper image of $l$ is a cubic curve passing through the basis points other than $i$, and having $l$ for bisecant.

\section{§2. Locus of Coincident Points.}

6. If $P^{\prime \prime}=P^{\prime}$, all the surfaces of the associated net touch each other at $P^{\prime}$, and one surface has a double point, or $P^{\prime}$ is the vertex of a cone belonging to (1). The equation of the locus of $P^{\prime}$ is expressed by the vanishing of the Jacobian of (1). It can be reduced to the form

$$
S_{4} \equiv\left|\begin{array}{llll}
a_{1} x_{2} x_{3} x_{4} & x_{1} & a_{1} & 1
\end{array}\right|=0
$$

The locus is the Weddle quartic surface. It contains $1,2, \ldots, 6$ as double points, the curve $\gamma_{3}$, the fifteen lines $(i k)$ and the ten lines of intersection of the planes $(i k l),(m n p)$; these lines $(i k l),(m n p)$ are defined by the symbol $(i k l)$. The four points of intersection of a line $l$ and its image $c_{7}$ lie on $S_{4}$, hence a general line $l$ contains no pair of associated points $\left(\xi^{\prime}\right),\left(\xi^{\prime \prime}\right)$. But it was seen that the lines joining $\left(\xi^{\prime}\right)$ to $\left(\xi^{\prime \prime}\right)$ are bisecants of $\gamma_{3}$, hence each of these bisecants contains an entire involution of associated points. The points on $\gamma_{3}$ constitute a pair and the remaining intersections with $S_{4}$ are the double points of the involution. The Weddle surface therefore cuts harmonically the bisecants of the cubic curve $\gamma_{3}$ which lies upon it.

7. In the involution $T_{3}$ of conjugate points as to $\gamma_{3}, S_{4}$ is invariant. Moreover, $I$ can now be defined independently of $(1)$ if the points $1,2, \ldots, 6$ are given, since $S_{4}$ and $\gamma_{3}$ are fixed. Given a point $P^{\prime}$ in space. Let the bisecant of $\gamma_{3}$ from $P^{\prime}$ cut $S_{4}$ in $M_{1}, M_{2}$. The harmonic conjugate of $P^{\prime}$ as to $M_{1}, M_{2}$ is $P^{\prime \prime}$. From the theorem of Stephanos * it follows that the transformations $T_{3}$ and $I$ are commutative.

\footnotetext{
* Mathematische Annalen, vol. 22 (1883), pp. 299-336. See page 313.
} 
8. A plane $\pi$ cuts its image $F_{7}$ in a curve of order seven and $S_{4}$ in a quartic which lies on $F_{7}$. The residual intersection consists of the three bisecants of $\gamma_{3}$ in $\pi$. A line cutting $\gamma_{3}$ goes into $c_{7}$ consisting of $\gamma_{3}$ and a rational quartic passing simply through all basis points and cutting the given line at the three residual intersections with $S_{1}$.

\section{§3. Transformations of $S_{4}$ into Itself.}

9. We may now enumerate the involutorial birational transformations of $S_{4}$ into itself :

The six nodal projections $\left(N_{1}\right)$;

The transformation $T_{3}$, defined by conjugate points as to $\gamma_{3}$;

The fifteen transformations defined by a secant of $\gamma_{3}$ and one of its bisecants $(i k)$. Given any point $P$ on $S_{4}$. The cubic cone projecting $\gamma_{3}$ from $P$ will be cut by the plane $P,(i k)$ in the two lines $P_{i}$ and $P_{k}$ and one other line. If the latter cuts $S_{4}$ in $Q$, between $P$ and $Q$ exists a birational correspondence. It will be designated by $T_{\text {yik }}$;

The interchange of points on every secant of two skew lines $T_{i k, l m}$ or $T_{i k, l m n}$;

The six transformations defined by the cubic curves passing through a point $P$ and five basis points. The residual point of intersection of the curve with $S_{4}$ is fixed when $P$ is known. We shall call these transformations $C_{i}, i$ being the symbol of the basis point through which the curve does not pass;

The transformations similar to the last preceding ones defined by the cubic curves passing through four basis points and having the line joining the remaining pair for bisecant. These transformations will be designated by $C_{i k}$;

An infinite number of further involutions can be defined as follows. Through any point on $\gamma_{3}$, a point of any fundamental line (ik) and a point $D$ on $S_{4}$ not on any fundamental line pass a quadric surface. It will belong to $(1)$, and cut $S_{4}$ in $\gamma_{3},(i k)$ and a space quartic passing through the remaining nodes. To get the point associated with any point $P$ on $S_{4}$, pass a plane through $P$ and any two nodes $l, m$ other than $i, k$. The plane cuts the space quartic in $l, m$ and two other points. Pass a conic through these four points on the quartic and through $P$. It is fixed, and will cut $S_{4}$ in one remaining point $Q$. The conjugate of this conic in $I$ is a sextic curve having double points at the basis points through which the conic does not pass, and passing simply through the six points in which the conic cuts $S_{4}$. The operation of interchanging $P, Q$ will be denoted by $X_{D}$. The same 
series of curves is invariant under $x$ as under $\left(N_{l}, N_{m}\right)$, but the former can be expressed in terms of the latter only when the line joining the points on the quartic is tangent to the curve elsewhere. But with the exception of the points $i, k$ this is impossible, since one point lies on $\gamma_{3}$ and the other on $(i k)$. The group generated by $X_{D}, X_{D^{\prime}}$, is of infinite order.

10. The points of $\gamma_{3}$ are defined in terms of the homogeneous parameters $\sigma, \tau$ by the equations

wherein

$$
\begin{aligned}
& \rho x_{1}=A \sigma \tau(\sigma-\tau), \\
& \rho x_{2}=B \sigma(\sigma-\tau)\left(\sigma_{1} \tau-\sigma \tau_{1}\right), \\
& \rho x_{3}=C \sigma \tau\left(\sigma_{1} \tau-\sigma \tau_{1}\right), \\
& \rho x_{4}=D \tau(\sigma-\tau)\left(\sigma_{1} \tau-\sigma \tau_{1}\right),
\end{aligned}
$$

$$
\begin{aligned}
& A=a_{1}\left(a_{2}-a_{3}\right)\left(a_{2}-a_{4}\right)\left(a_{4}-a_{3}\right), \quad B=a_{2}\left(a_{4}-a_{3}\right), \quad C=a_{3}\left(a_{2}-a_{4}\right), \\
& D=a_{4}\left(a_{2}-a_{3}\right), \quad \sigma_{1}=\left(a_{2}-a_{3}\right)\left(a_{1}-a_{4}\right), \quad \tau_{1}=\left(a_{1}-a_{2}\right)\left(a_{4}-a_{3}\right) .
\end{aligned}
$$

The values of the parameters at the basis points are :

$$
\begin{array}{lll}
\text { at } 1, & \sigma=\left(a_{2}-a_{3}\right)\left(a_{1}-a_{4}\right), & \tau=\left(a_{1}-a_{2}\right)\left(a_{4}-a_{3}\right), \\
\text { at } 2, & \sigma=a_{2}-a_{3}, & \tau=a_{4}-a_{3}, \\
\text { at } 3, & \sigma=1, & \tau=0, \\
\text { at } 4, \quad \sigma=a_{4}\left(a_{2}-a_{3}\right), & \tau=a_{2}\left(a_{4}-a_{3}\right), \\
\text { at } 5, \quad \sigma=1, & \tau=1, \\
\text { at } 6, & \sigma=0, & \tau=1 .
\end{array}
$$

These equations may be used in $C_{2}$ by replacing $(a)$ by the coördinates of $P$. The other $C_{i}$ can be obtained from it by linear transformations.

The equations of the Cremona transformation $T_{3}$ can be obtained from the preceding equations alone.

\section{§4. Special Positions of Basis Points.}

11. The quadric surface determined by the lines (12), (35), (46) has the equation

$$
x_{4}\left[x_{1}\left(a_{2}-a_{3}\right)+a_{3} x_{2}-a_{2} x_{3}\right]+x_{1}\left[a_{4} x_{3}-a_{4} x_{2}\right]=0 .
$$

It cuts $\gamma_{3}$ in the six basis points; if upon the coefficients the condition is 
imposed that one further point of $\gamma_{3}$ lies on the quadric, then $\gamma_{3}$ lies entirely upon it. This condition is

$$
a_{2}\left(a_{1}-a_{3}\right)=a_{1}\left(a_{4}-a_{3}\right) .
$$

In this case the four nets $\left\{\gamma_{3}\right\},\{12\},\{35\},\{46\}$ have a common quadric.

The intersection of a quadric with $S_{4}$ consists of a curve of order eight cutting each generator in both reguli in four points. On one regulus the points of intersection are on the three lines (12), (35), (46) and on $\gamma_{3}$; on the other, two of them are on $\gamma_{3}$, hence the residual intersection consists of a curve of order two cutting the generators of the second regulus in two points and not cutting those of the first, that is, it is composed of two straight lines $u, v$.

The equations of these lines are

$$
l x_{1}-m x_{4}=0, \quad x_{1}\left(a_{2} l-a_{3} l\right)+x_{2}\left(a_{3} l-a_{4} m\right)+x_{3}\left(a_{4} m-a_{2} l\right)=0,
$$

the parameters $l: m$ being defined by the quadratic equation

$$
a_{1} l^{2}-2 a_{1} l_{m}+a_{4} m^{2}=0 \text {. }
$$

If the points $1,2, \cdots, 6$ are projected from any point of $\gamma_{3}$ upon a plane, the generator of the same regulus as $u$, $v$ which passes through the center of projection will pierce the plane of projection in a point through which pass the projections of (12), (34), (56). The basis points thus project into six points in involution. Conversely, when from any point of $\gamma_{3}$ these points are projected into points in involution, they and $\gamma_{3}$ have a common transversal and the preceding condition applies. We shall speak of the six points $1,2, \ldots, 6$ themselves as being in involution when their projections are in involution.

When the six basis points $1,2, \ldots, 6$ are in involution the surface $S_{4}$ contains two new lines $u, v$. The generators of the regulus cutting $u, v$ are bisecants of $\gamma_{3}$, cutting $S_{4}$ in the residual points on $u, v$. These four points of intersection are harmonic. In the axial involution determined by $u, v$ the six points $1,2, \ldots, 6$ are interchanged in pairs and $\gamma_{3}$ goes into itself, hence $S_{4}$ must remain invariant. In this case the axial involution $I_{1}$ must be added to the preceding transformations.

12. In the same manner it is found that (13), (24), (56) are in involution if the $a_{i}$ satisfy the condition $a_{1} a_{2}-a_{3} a_{4}=0$;

$$
\begin{aligned}
& \text { (16), (34), (25) are in involution if } a_{1}\left(a_{3}-a_{4}\right)=a_{3}\left(a_{2}-a_{4}\right) \text {; } \\
& \text { (12), (36), (45) " " " " } \quad \text { " } a_{1}\left(a_{3}-a_{4}\right)=a_{2}\left(a_{1}-a_{4}\right) \text {; } \\
& (14),(23),(56) \quad \text { " " } \quad \text { " } \quad a_{2}\left(a_{4}-a_{1}\right)=a_{3}\left(a_{4}-a_{2}\right) \text {; } \\
& \text { (15), (26), (34) “ " " } \quad \text { " } a_{1}\left(a_{4}-a_{3}\right)=a_{4}\left(a_{2}-a_{3}\right) \text {. }
\end{aligned}
$$


These six conditions are all satisfied when the $a_{i}$ have the values

$$
a_{1}=1, \quad a_{2}=2, \quad a_{3}=1+i, \quad a_{4}=1-i .
$$

The surface $S_{4}$ now has six pairs of lines $u_{i}, v_{i}$ and is invariant under six axial involutions $I_{i}$. No two of these involutions can be commutative. In order that two axial involutions may be commutative it is necessary that their axes shall either intersect or belong to the same regulus. Let $(u, v),\left(u^{\prime}, v^{\prime}\right)$ be the axes and suppose $u, u^{\prime}$ intersect in $M$. The plane of $u, u^{\prime}$ cuts $v$ in $K$, $v^{\prime}$ in $L$. Any line through $K$ cuts $S_{4}$ in a fourth point which is the harmonic conjugate of its intersection with $u^{\prime}$ as to its intersection with $u$ and $K$. The locus of this point is a line through $M$. The residual intersection of the plane consists of another line, this latter passing through $K$. But this is impossible, as $S_{4}$ cannot contain so many coplanar lines.

If $(u, v),\left(u^{\prime}, v^{\prime}\right)$ belong to the same regulus, they must be harmonic, since any transversal cuts $S_{4}$ only on these lines. The quadric determined by them would cut $S_{4}$ in four lines belonging to the other regulus, of which one at most can be a line $(i k)$. Of the six lines $(i l m)$ skew to $(i k)$, three can be chosen that are mutually skew and do not have another fundamental line for common transversal. Since $u, v, u^{\prime}, v^{\prime}$ are all invariant under $I$, a generator of the same regulus goes into a sextic cutting every generator of each system in three points. But this curve has double points at the fundamental points except $i$ and $k$, hence all the fundamental points lie on the quadric, which therefore belongs to the system $(1)$. Since $(l m)$ cuts $u, v$, it must therefore lie on the quadric, but this is impossible, as the entire intersection with $S_{4}$ has been accounted for. Hence in this case $u, v, u^{\prime}, v^{\prime}$ cannot belong to the same regulus. Similarly for the case when no line $(i k)$ cuts both pairs of axes. This completes the proof that no two operations $I_{i}, I_{k}$ are commutative.

\section{§5. Double ג-Space.}

In (1) let the $\lambda_{i}$ be considered as homogeneous plane coördinates. The plane will define a quadric of the system (1). A linear equation will define a point in $(\lambda)$. This equation and (1) will determine a net of quadrics, passing through $1,2, \ldots, 6$ and two associated points $\left(\xi^{\prime}\right),\left(\xi^{\prime \prime}\right)$. If the coördinates of $\left(\xi^{\prime}\right)$ are substituted in (1), the resulting equation will fix the same point in $(\lambda)$. Thus, the equation $(1)$ defines a $(1,2)$ correspondence between the points $(\lambda)$ of a space $\Sigma^{\prime}$ and the points $\left(\xi^{\prime}\right),\left(\xi^{\prime \prime}\right)$ of another space $\Sigma$. Two linear equations in $(\lambda)$ define a straight line in $\Sigma^{\prime}$. From these equations and (1) a pencil of quadrics is fixed, whose basis curve passes through the basis points $1,2, \ldots, 6$. Hence when $\left(\xi^{\prime}\right)$ describe a plane in $\Sigma,(\lambda)$ will describe a quartic surface. The complete image in $\Sigma$ of this quartic surface in $\Sigma^{\prime}$ is the plane and its image $F_{7}$ 
in $I$. To a straight line in $\Sigma$ corresponds a conic in $\Sigma^{\prime}$, whose complete image in $\Sigma$ is the line and its image $c_{n}$.

If in the first derivatives of $(1)$ we eliminate $x_{i}$, the resulting equation of order four in $\lambda_{i}$ will define those values for which $\left(\xi^{\prime}\right) \equiv\left(\xi^{\prime \prime}\right)$. They define a surface of class four, $\Delta(\lambda)$. If $\Sigma^{\prime}$ is regarded as a double space, one sheet corresponding to $\left(\xi^{\prime}\right)$, the other to $\left(\xi^{\prime \prime}\right)$, the points of $\Delta(\lambda)$ are branch points. The points of $\Delta(\lambda)$ and the points of $S_{4}$ are in $(1,1)$ correspondence. Since an arbitrary line in $\Sigma$ is not its own image in $I$, the image conies cannot intersect $\Delta(\lambda)$, hence :

The images of the $\infty^{4}$ lines of $\Sigma$ are $\infty^{4}$ conics which touch four tangent planes of $\Delta$.

The image of a general plane $\pi$ touches $\Delta$ along a curve of order eight. To a plane section of $\Delta$ corresponds a curve of order eight, in which the image quadric cuts $S_{4}$. A tangent plane of $\Delta$ corresponds to a cone of $\Sigma$, belonging to (1). To the point of contact corresponds its vertex. To the four cones in a pencil of quadrics belonging to (1) correspond the four tangent planes through the image line of the basis quartic.

14. To an arbitrary point $\Sigma a_{i} \lambda_{i}$ corresponds a pair of points $\left(\xi^{\prime}\right),\left(\xi^{\prime \prime}\right)$. The $\infty^{2}$ planes of $\Sigma^{\prime}$ through this point correspond to the $\infty^{2}$ quadrics through $1,2, \ldots, 6,\left(\xi^{\prime}\right),\left(\xi^{\prime \prime}\right)$. In particular, to the tangent planes to $\Delta$ passing through the point correspond $\infty^{1}$ cones in the net of quadries. The locus of the vertices of these cones is a curve of order eight on $S_{4}$, having double points at all eight basis points. If in $\Sigma$ we project the $\infty^{2}$ basis curves of order four and this octic from one of the basis points, and take the dual of the configuration in $\Sigma^{\prime}$ as to a quadric surface, we have exactly the Geiser transformation which was discussed in a preceding paper.*

Let $P_{00}^{\prime}$ in $\Sigma^{\prime}$ correspond to the net of quadrics $\left\{\gamma_{3}\right\}$. It is a principal point of $\Sigma^{\prime}$. Similarly, let $P_{i k}^{\prime}$ correspond to $\{i k\}$. It is also a principal point. Any plane $\pi$ in $\Sigma$ cuts $\gamma_{3}$ in three points and each of the fifteen lines ( $\left.i k\right)$ once. Its image $F^{\prime}$ in $\Sigma^{\prime}$ passes three times through $P_{00}^{\prime}$ and once through each of the fifteen points $P_{i k}^{\prime}$. Since $\gamma_{3}$ and $(i k)$ all lie on $S_{4}, P_{00}^{\prime}, P_{i k}^{\prime}$ all lie on $\Delta$. The $c_{8}^{\prime}$ of contact of $\Delta$ and $F_{4}^{\prime}$ passes three times through $P_{00}^{\prime}$ and simply through $P_{i k}^{\prime}$.

Every bisecant of $\gamma_{3}$ contains a whole involution of points $\left(\xi^{\prime}\right),\left(\xi^{\prime \prime}\right)$. Its image in $\Sigma^{\prime}$ is a straight line through $P_{\mathrm{v} 0}^{\prime}$, counted twice. The bundle of lines through $P_{00}^{\prime}$ in $\Sigma^{\prime}$ and the $(1,3)$ congruence of bisecants of $\gamma_{3}$ are in $(1,1)$ correspondence.

Since $\pi$ cuts $\gamma_{3}$ in three points and contains three bisecants, $F_{4}^{\prime}$ contains three double lines passing through $P_{00}^{\prime}$. Thus $F_{4}^{\prime}$ is a Steiner surface. A line $l$ in $\pi$ corresponds to a conic $c_{2}^{\prime}$ on $F_{4}^{\prime}$. The plane $\omega^{\prime}$ of $c_{2}^{\prime}$ corresponds to a

\footnotetext{
* SNyder : Conjugate line congruences contained in a bundle of quadric surfaces, these Transactions, vol. 11 (1910), pp. 371-387.
} 
quadric $H$ containing $l$ and another line $m$ lying in $\pi$; the image of $m$ is a second conic $k_{2}^{\prime}$ in $\omega^{\prime}$ on $F_{4}^{\prime}$. These two conics intersect on the three double lines and in one other point, the image of $l m$. $H$ touches $\pi$ in $l m$; $\omega^{\prime}$ touches $F_{4}^{\prime}$ in $c_{2}^{\prime}, k_{2}^{\prime}$. Both of these conics are contact curves of $\Delta$.

15. The points $1,2, \ldots, 6$ are principal points of the transformation; the image of each is a plane $\pi_{i}^{\prime}$. All the basis points lie on $\gamma_{3}$, hence the six planes $\pi_{i}^{\prime}$ pass through $P_{00}^{\prime}$; $(i k)$ passes through two, hence $\pi_{i}^{\prime}, \pi_{k}^{\prime}$ pass through $P_{i k}^{\prime}$. The fifteen lines $P_{00}^{\prime} P_{i k}^{\prime}$ are therefore the intersections of the six planes $\pi_{i}^{\prime}, \pi_{k}^{\prime}$. Since a plane in $\Sigma^{\prime}$ is the image of a quadric in $\Sigma$, the planes $\pi_{i}^{\prime}$ may be regarded as images of the cones in (1), having $i$ for vertex and passing through the other basis points. A bisecant of $\gamma_{3}$ cuts $S_{4}$ in two points not on $\gamma_{3}$; its image cuts $\Delta$ in two points not at $P_{00}^{\prime}$.

A basis $c_{4}$ in $\Sigma$ cuts $S_{4}$ in four points besides the basis points, hence its image line cuts $\Delta$ in four points. $\Delta$ is of order four. The surface of branch points in $\Sigma^{\prime}$ is of order and class four and has sixteen double points $P_{00}^{\prime}, P_{i k}^{\prime}$, hence it is a Kummer surface. To a straight $l^{\prime}$ through $P_{i k}^{\prime}$ corresponds a quartic curve in $\Sigma$ consisting of $(i k)$ and a cubic curve cutting ( $i k)$ twice and passing through the other four basis points. The curve cuts $S_{4}$ in two points not at basis points nor on $(i k)$, hence its image line cuts $\Delta$ in two points not at $P_{i k}^{\prime}$, or $P_{i k}^{\prime}$ is also a double point.

16. Any line $l^{\prime}$ in $\pi_{i}^{\prime}$ goes into a $c_{4}$ on $K_{2}^{(i)}$, but as this fundamental cone is the tangent cone to $S_{4}$ at $i, c_{4}$ has a double point at its vertex, and all the variable points of intersection with $c_{4}, S_{4}$ are at $i$, two on each nodal tangent. Thus $l^{\prime}$ is a double tangent to $\Delta$, or $\pi_{i}^{\prime}$ contains a conic of contact. It passes through $P_{00}^{\prime}$ and $P_{i k}^{\prime},(k=1,2, \ldots, 6, i \neq k)$.

The plane (123) goes into an $F_{4}^{\prime}$ consisting of $\pi_{1}^{\prime}, \pi_{2}^{\prime}, \pi_{3}^{\prime}$ and another plane $\pi_{123}^{\prime}$, whose complete image in $\Sigma$ consists of (123) and its image in $I$, viz., (456). The line (123), (456) goes into the conic of contact of $\pi_{123}^{\prime}$. The principal points lying upon it are $P_{12}^{\prime}, P_{13}^{\prime}, P_{23}^{\prime}, P_{45}^{\prime}, P_{46}^{\prime}, P_{56}^{\prime}$. The distribution of singular points and singular planes can now be readily determined by observing the corresponding elements in $\Sigma$.

A line $l$ through any basis point $i$ in $\Sigma$ corresponds to a line in $\Sigma^{\prime}$, for, if any quadric $H$ of (1) corresponds to the plane $p^{\prime}, l$ cuts $H$ in as many variable points and the image cuts $p^{\prime}$. But $H$ passes through $i$, hence $l$ corresponds to a straight line $l^{\prime}$. Since $l$ does not correspond to itself in $I, l^{\prime}$ is a bitangent of $\Delta$. Thus the bitangents of $\Delta$ are arranged in six distinct systems corresponding to the six basis points $1,2, \ldots, 6$, in $\Sigma$.

\section{§6. Image Congruences of the Bundles $1,2, \ldots, 6$.}

17. Through any point $P^{\prime}$ in $\Sigma^{\prime}$ pass two lines of the congruence [i], since $P^{\prime}$ has two image points $\left(\xi^{\prime}\right),\left(\xi^{\prime \prime}\right)$ in $\Sigma$, each of which defines a line of $i$. A 
quadric $H$ of (1) contains two lines of $i$, hence its image plane contains two lines of [i]. Any line $l$ of $i$ cuts $S_{4}$ in two residual points. These go into the points of contact of $l^{\prime}$ with $\Delta$. The complete image in $\Sigma$ of $l^{\prime}$ consists of $l$ and its image in $I$, that is, a space cubic curve passing through the remaining basis points and cutting $l$ at its residual intersection with $S_{4}$.

The arrangement of the lines of these congruences can now be ascertained. The bundle $i$ can be considered as made up of a single infinity of plane pencils each in a plane through $(i k)$. The image of each plane is a Steiner surface $F_{4}^{\prime}$ consisting of $\pi_{i}^{\prime}$ and $\pi_{k}^{\prime}$ and a quadric surface. The generators of one regulus are the images of the lines of the pencil $i$, and those of the other regulus are images of the lines through $k$. These quadrics all pass through eight basis points and form a singly infinite quadratic system. Their envelope is the surface $\Delta .^{*}$

\section{§7. Depiction of $S_{4}$ on $\Delta$.}

18. We can now study the transformations which leave $\Delta$ invariant by depicting $S_{4}$ upon $\Delta$ and considering the transformations into which those enumerated in No. 9 are transformed. From the preceding section it follows immediately that the nodal projections $\left(N_{i}\right)$ become those of interchanging the points of contact on the lines of congruences $[i] . \dagger$

The six operations $[i]$ generate an abelian group of order 32 . The product of any two of them is an axial homology. By No. 14 the operation $T_{3}$ of conjugate points as to $\gamma_{3}$ becomes the nodal projection $\left(P_{00}^{\prime}\right)$ of $\Delta$ into itself from $P_{00}^{\prime}$. The involution $I$ is transformed into a Jonquières transformation leaving every point of $\Delta$ invariant. To find the image of any point $A^{\prime}$, connect it with $P_{00}^{\prime}$. The line $A^{\prime} P_{00}^{\prime}$ cuts $\Delta$ in $M_{1}^{\prime}, M_{2}^{\prime}$. The harmonic conjugate of $A^{\prime}$ as to $M_{1}^{\prime} M_{2}^{\prime}$ is the required image.

19. In $\Sigma^{\prime}$, the nodal projection $\left(P_{i k}^{\prime}\right)$ can be obtained by transforming $\left(P_{00}^{\prime}\right)$ through $[i][k]$,

$$
\left(P_{i k}^{\prime}\right)=[i][k]\left(P_{00}^{\prime}\right)[k][i] .
$$

In $\Sigma$ this operation requires that a point be projected from $i$, its image from $k$, this image found as to $\gamma_{3}$, then the first two operations repeated in reverse order Pass two quadrics $H_{1}, H_{2}$ of the system (1) through the line (ik). Their

* The equations of these quadrics, the configuration of the associated basis points, and all the properties of the congruences of order and class two are at once derived by projecting the cubic variety having 10 nodes in four way space from a point upon it, as is outlined in my paper : Surfaces derived from the cubic variety having nine double points in four dimensional space, these Transactions, vol. 10 (1909), pp. 71-78. In the notation of that paper, $\lambda=1$ will define as contour a general Kummer surface.

†This result was first found by $H$. F. BAKER by another method. A different proof is given in my paper : Infinite discontinuous groups of birational transformations which leave certain surfaces invariant, these Transactions, vol. 11 (1910), pp. 15-24. See page 17 for further references. 
residual intersection consists of a cubic passing through the other basis points, and cutting ( $i k)$ twice. The cubic is fixed by any point $P$ on $S_{4}$. The images in $\Sigma^{\prime}$ of $H_{1}, H_{2}$ are two planes each passing through $P_{i k}^{\prime}$. Their intersection consists of a straight line which is fixed by $P^{\prime}$, the image of $P$.

The fifteen operations $C_{i k}$ are transformed into the fifteen nodal projections $\left(P_{i k}^{\prime}\right)$.

$$
C_{i k}=\left(N_{i}\right)\left(N_{k}\right) T_{3}\left(N_{k}\right)\left(N_{i}\right) \text {. }
$$

20. Any two of these operations generate a group of infinite order. Moreover, the index of this group as to that generated by two pairs will also be infinite. This can be most easily proved in $\Sigma^{\prime}$. Let $\left(P_{12}^{\prime}\right)\left(P_{34}^{\prime}\right)=s$ and $\left(P_{15}^{\prime}\right)\left(P_{36}^{\prime}\right)=t$.

Pass a plane through $P_{12}^{\prime} P_{34}^{\prime}$ and any point $P^{\prime}$ on $\Delta$. This plane cuts $\Delta$ in a general quartic curve having two nodes. When $P^{\prime}$ is operated upon by $s$, its image will always lie on the same quartic curve. Pass a plane through $P^{\prime}$, $P_{15}^{\prime}, P_{36}^{\prime}$. It will cut $\Delta$ in a quartic curve which intersects the preceding one in three points $A_{1}^{\prime}, A_{2}^{\prime}, A_{3}^{\prime}$ besides $P^{\prime}$. The images of $P^{\prime}$ when operated upon by $t$ will lie in the second plane. If any power of $s$ were equal to any power of $t$, the corresponding position of $P^{\prime}$ would be at $A_{i}^{\prime}$, hence these respective operations repeated four times would cause the point to return to $P^{\prime}$. If the period is finite for one point it has been proved to be finite for every point, but $s$ and $t$ are each of infinite order. Hence no relation of the form $s^{m}=t^{n}$ can be found.

Now multiply the series $1, s, s^{2}, s^{3} \ldots$ by $t, t^{2}, \cdots$ on the right. If $\mathrm{s}^{k} t^{l}=s^{p} t^{q}$, then $s^{k-p}=t^{q-l}$, which relation was proved to be impossible. It follows that no operation $s^{k} t^{l}$ appears twice in this set, or that the index of the group generated by $s$ and $t$ is of infinite order as to the group generated by $s$. In the same manner we can obtain a group generated by $s, t, u$, having an infinite index as to that generated by $s, t$, etc. All the operations of the group can be expressed in terms of $\left(P_{00}^{\prime}\right)$ and the operators of the linear group $G_{16}$.

21. Since the operations $C_{i}$ and $\left(N_{i}\right)$ are transformed into each other by $I$, it follows that $C_{i}$ goes into $[i]$ in $\Sigma^{\prime}$.

22. The straight lines defining $T_{y i k}$ go into conics in $\Sigma^{\prime}$ passing through $P_{00}^{\prime}$ and $P_{i k}^{\prime}$. Since the lines in $\Sigma$ do not go into themselves when operated upon by $I$, it follows that the image conics in $\Sigma^{\prime}$ touch $\Delta$ in the two points which are images of the residual points of intersection of the line and $S_{4}$. Through any point $P$ of $S_{4}$ can be drawn but one line cutting $\gamma$ and $(i k)$; it will cut $S_{4}$ in $Q$. The basis curve composed of $\gamma$ and $(i k)$ will define a pencil of quadric surfaces, one of which passes through $P$. This quadric goes into the plane passing through $P_{00}^{\prime}$ and $P_{i k}^{\prime}$, cutting $\Delta$ in a binodal quartic curve. Through $P^{\prime}$ can be passed four conics, all of which touch $\Delta$ at $P^{\prime}$, pass through $P_{00}^{\prime}, P_{i k}^{\prime}$, and touch $\Delta$ in another point. The generators of the other regulus on the quadric in $\Sigma$ go into $\infty^{1}$ conics which are proper contact conics, that do not pass through 
the nodes. Any binodal quartic can be generated in four ways as the envelope of a conic passing through the nodes; its pole as to the line joining them describes a conic. The four loci of poles are inscribed in the same quadrilateral, having a vertex at each node. The series of conics are rationally separated when one position of the pole is known. The second point of contact of one conic is $Q^{\prime}$. When $P^{\prime}$ describes $\Delta, Q^{\prime}$ can be distinguished from the other points of contact by means of the equation of the locus of the pole. Each of the other series of contact conics will also define birational transformations, but these are not images of straight lines in $\Sigma$. The conjugate operation in $I$ is defined by the cubics passing through four basis points and cutting $\gamma$ in one variable point.

In the same way, the operations $T_{i k, l m}$ are transformed into the operations defined by the series of contact conics passing through $P_{i k}^{\prime}, P_{l m}^{\prime}$. They are obtained from the preceding series by the linear transformations [i] $[k]$. Now consider the lines (123), (14) in $\Sigma$. The former goes into a conic through $P_{12}^{\prime}, P_{13}^{\prime}, P_{23}^{\prime}, P_{45}^{\prime}, P_{46}^{\prime}, P_{56}^{\prime}$, or the singular conic in the plane $\pi_{123}^{\prime}$. The line (14) goes into the point $P_{14}^{\prime}$. Any line in $\Sigma$ cutting (123), (14) goes into a conic in $\Sigma^{\prime}$ passing through $P_{14}^{\prime}$, touching the conic of contact in the singular plane $\pi_{123}^{\prime}$, and touching $\Delta$ in the images of the residual intersections of the line and $S_{4}$. As before, three extraneous series of conics appear.

23. The conjugate of the lines cutting $(i k),(l m)$ in $I$ are quintic curves through $i, k, l, m$ simply, through the remaining basis points twice, having one variable point on $(i k)$ and on $(i l)$. They are uniquely fixed by a point $P$ on $S_{4}$ and pass through $Q$, the residual point of intersection of the line through $P$ outting $(i k),(l m)$. As in the case of the cubics $C_{i}$, these curves could be used to define the transformation. Whenever the corresponding transformation in $\Sigma^{\prime}$ is defined by rational contact curves, two distinct systems of rational curves in $\Sigma$ will define the same transformation of points on $S_{4}$. On the other hand a Cremona transformation in $\Sigma^{\prime}$ may not be expressible in rational form in $\Sigma$, except for points of $S_{4}$.

24. To obtain the operation into which $X$ is transformed in $\Sigma^{\prime}$, it will be observed that the quadric passing through $\gamma_{3}$ and $(i k)$ goes into a plane, and the section of the former with $S_{4}$ becomes a plane quartic curve, section of $\Delta$, having nodes at $P_{00}^{\prime}$ and $P_{i k}^{\prime}$. This section is fixed when one point upon $\Delta$ is given.

Through the conic passing through $l, m$ can be passed a quadric belonging to (1), hence its image in $\Sigma^{\prime}$ is a plane. The plane of the conic is transformed into a Steiner surface having $\pi_{l}^{\prime}, \pi_{m}^{\prime}$ as factors, hence a quadric. The generators of one regulus are the images of the pencil of lines through $l$ in the plane of the conic, and those of the other are images of the lines through $m$. The image of the conic is therefore a conic. On $\Sigma$, the space quartic passes through all the basis points except $i$ and $k$. A plane through any other two will cut the curve in but two other points. The image quadric will therefore have but two points in common 
with the image plane curve, that is, the conic in the plane of section will touch the curve at the two points. Through any point in the section of the quadric with $\Delta$ can be drawn a conic touching the curve which its plane cuts from $\Delta$ at two fixed points, the chosen point $P^{\prime}$ and at one other point $Q^{\prime}$. This is the transform of the operation $X$.

\section{§8. The Tetrahedroid.}

25. When the points $(1,2),(3,5),(4,6)$ are in involution, it was seen that the nets $\left\{\gamma_{3}\right\},\{12\},\{35\},\{46\}$ have a quadric in common. This quadric becomes a plane in $\Sigma^{\prime}$ passing through the four points $P_{00}^{\prime}, P_{12}^{\prime}, P_{35}^{\prime}, P_{46}^{\prime}$. Since the section of $\Delta$ made by the plane is a quartic having four double points, the curve of section consists of two conics. By the sixteen linear operations which leave $\Delta$ invariant the plane of the four nodes can be transformed into three others, forming with the given one a tetrahedron. By the operations [i], the dual configuration is obtained, such that four points exist through each of which pass four of the singular planes of $\Delta$. These planes intersect in six lines, on each of which lie two nodes, hence the nodes on any singular conic are in involution.

If the six nodes of $S_{4}$ are in involution, the six nodes in every singular conic of $\Delta$ are in involution. $S_{4}$ now has an extra pair of lines and $\Delta$ has four pairs of conics.

26. The axial involution in $\Sigma$ goes into the operation defined by interchanging the points of contact of a conic which touches $\Delta$ in the images of the axes of the involution and in two other points. The four points of contact are harmonic. $\Delta$ is now a tetrahedroid; since the lines joining two nodes define an involution in each singular plane, and four singular planes pass through the center of this involution, $\Delta$ is now invariant under the central involutions defined by the vertices and opposite faces of the tetrahedron formed by the planes containing pairs of conics on $\Delta$. These operations cannot be defined in terms of the axial involutions in $\Sigma$. Let $P^{\prime}$ be the center and $\pi^{\prime}$ the invariant plane. The image of $P^{\prime}$ in $\Sigma$ is a pair of points $\xi^{\prime}$, $\xi^{\prime \prime}$ not lying on $S_{4}$; the image of $\pi^{\prime}$ is the quadric containing $u, v$. A line through $P^{\prime}$ cuts $\pi^{\prime}$ in $\Pi^{\prime}$ and $\Delta$ in two pairs of points $M_{i}^{\prime}$, each harmonic with $P^{\prime} \Pi^{\prime}$. The image of the line is a quartic curve passing through the eight associated points $1,2, \cdots, 6, \xi^{\prime}, \xi^{\prime \prime}$. It cuts the quadric in two points $\eta^{\prime}, \eta^{\prime \prime}$ and $S_{4}$ in four points, images of $M_{i}^{\prime}$.

27. When the points $1, \ldots, 6$ are in multiple involution, $\Delta$ is a multiple tetrahedroid. In the case of six fold involution the new central involutorial perspective transformations and the linear $G_{16}$ generate a group of order 24.16.*

\footnotetext{
* Segre, Sur un cas particulier de la surface de Kummer, Leipziger Berichte, vol. 36 (1884), pp. 132-135.
} 